\title{
Editorial Referateband 2019
}

\section{Digitalisierung - Herausforderung für unser Fachgebiet}

\section{(ㄷ) (1) $(2) \ominus$}



Prof. Dr. med. Stefan Dazert
Korrespondenzadresse

Prof. Dr. med. Stefan Dazert

Präsident der Deutschen Gesellschaft für HNO-Heilkunde

Kopf- und Hals-Chirurgie e.V. 2018/2019

Univ. HNO-Klinik, St. Elisabeth-Hospital

Bleichstr. 15

D-44787 Bochum

Bibliografie

DOI https://doi.org/10.1055/a-0740-4470

Laryngo-Rhino-Otol 2019; 98: S1-S2

(c) Georg Thieme Verlag KG Stuttgart · New York

ISSN 0935-8943
Liebe Kolleginnen, liebe Kollegen, es freut mich außerordentlich, den aus meiner Sicht sehr spannenden Referateband zur 90. Jahresversammlung der Deutschen Gesellschaft für Hals-Nasen-Ohren-Heilkunde, Kopf- und Hals-Chirurgie vorzustellen. Die hochinteressanten wissenschaftlichen Abhandlungen aus neun Universitäten bearbeiten unterschiedliche Bereiche zum Kongressthema „Digitalisierung in der HNO-Heilkunde" und zeigen wichtige Aspekte für die Weiterentwicklung und Zukunftssicherung unseres Fachgebietes auf.

Während der letzten Jahre gewinnen Digitalisierung und künstliche Intelligenz in der deutschen Gesundheitslandschaft und somit auch für die HNO-Heilkunde zunehmend an Bedeutung. Im Hinblick auf zukünftige Entwicklungen in der Gesundheitsversorgung ist es erforderlich, digitale Kompetenzen sowohl Ärzten/-innen, medizinischem Fachpersonal aber auch Patienten/-innen strukturiert zu vermitteln. Ebenso müssen die sich aus den medizinischtechnischen Fortschritten ergebenden rechtlichen und ethischen Fragen diskutiert werden.

Die Digitalisierung hält in alle gesellschaftlich relevanten Bereiche rasant Einzug und bestimmt die globale wirtschaftliche Wertentwicklung entscheidend, wie an den großen, international agierenden Datenkonzernen abzulesen ist. Die digitale Transformation unserer Gesellschaft ist somit in vollem Gange und erreicht natürlich zunehmend auch das Gesundheitswesen. Im internationalen Vergleich liegen deutsche Gesundheitseinrichtungen im Hinblick auf digitale Entwicklungen eher auf hinteren Rängen, sodass sofortige und intensive Anstrengungen in diesen Themenfeldern zwingend erforderlich sind.

Die in diesem Band vorgestellten Referate greifen wichtige Bereiche der Digitalisierung im Gesundheitswesen auf, denen für die zukünftige Entwicklung des HNO-Faches eine entscheidende Bedeutung zugeschrieben wird.
So werden die Grundlagen der elektronisch-medizinischen Datenhaltung bearbeitet, die bereits in verschiedene Gesundheitsbereiche Einzug gehalten hat. Insbesondere die elektronische Patientenakte steht im Sinne einer höheren Versorgungsqualität bei geringeren Versorgungskosten im Fokus des Interesses. Die Registerbildung hat die Evaluation der Wirksamkeit in der Versorgungsroutine, das Monitoren der Patientensicherheit sowie die ökonomische Evaluation und die Mindestmengenforschung zur Aufgabe. Weiterhin verfolgen Register das Ziel, epidemiologische Zusammenhänge und Unterschiede zu beschreiben, die Qualitätssicherung und -verbesserung sowie die klinische Forschung zu unterstützen.

Im Kontext mobiler Informations- und Kommunikationstechnologie für medizinische Dienstleistungen, steht auch bei der Anwendung von Suchmaschinen und Apps die Qualitätsfrage im Vordergrund. So werden die Rolle des Internets für die stetig zunehmende Bereitstellung von Gesundheitsinformationen beleuchtet und wichtige Hinweise zur Qualität der verfügbaren Daten für den Nutzer gegeben. Für Anwender medizinischer Apps werden in einem weiteren Beitrag Möglichkeiten vorgestellt, diese Applikationen nach definierten Qualitätskriterien in verschiedenen Kategorien, wie z. B. Funktionalität, Wissenschaftlichkeit, aber auch Datenschutz, zu beurteilen und nutzbringend einzusetzen.

Als Beispiel einer unmittelbaren Anwendung für die digital unterstützte Patientenversorgung im Krankenhaus, kann die Etablierung eines „digitalen (HNO-) OP-Saals der Zukunft“ angesehen werden. Hier geht es nicht nur um die rein technische Verbesserung der einzelnen computergestützten Geräte und Instrumente, sondern vielmehr um deren dynamische Vernetzung und Systemintegration in einem offenen modularen System mit dem Ziel, die Anwenderfreundlichkeit, die Qualität und die Patientensicherheit zu verbessern. 
Ähnliche Zielsetzungen im Hinblick auf die Versorgungsqualität ergeben sich für die digitalen Entwicklungen der Telemedizin in unserem Fachgebiet sowie für moderne Konzepte der Hörrehabilitation, wo der Einsatz von artificial intelligence und remote care neue Strategien eröffnet. Die molekulargenetische Diagnostik von Hörstörungen in einem Krankheitskontext basiert heute wesentlich auf der sich rasch entwickelnden Genomik, die auf technologische Entwicklungen in der Sequenzierung, Computer- und Bioinformatik zurückzuführen ist. Der Begriff big data spielt hier eine entscheidende Rolle und beinhaltet die Verwendung großer Datenmengen, die in Form von genetischen Datenbanken, in silico Analysewerkzeugen und Allel-Häufigkeitsdatenbanken vorliegen.

Die Entwicklung neuer digitaler Strategien in der Lehre beruht auf den technologischen Fortschritten der letzten Jahrzehnte, aber v. a. auf der Motivation, didaktische Konzepte verstärkt auf den Lernenden zu zentrieren. Der Digitalisierungsgrad der Lehre reicht heute von digitalen Serviceangeboten über punktuelle digitale Angebote klassischer Lehrkonzepte bis hin zum volldigitalen Studium. Hier werden für die HNO-Heilkunde relevante Aspekte der auf die heutigen Bedürfnisse der Studierenden ausgerichteten Lehrkonzepte vorgestellt.

Ich wünsche Ihnen eine interessante Lektüre mit hoffentlich vielen Anregungen für die zukünftige tägliche Arbeit in Klinik und Praxis. Mit einem herzlichen Glück auf, dem Gruß aus dem Ruhrgebiet, Ihr

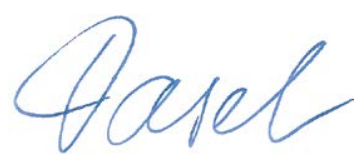

Prof. Dr. med. Stefan Dazert

Präsident der Deutschen Gesellschaft für HNO-Heilkunde, Kopf- und Hals-Chirurgie 2018/2019 